\title{
Emerging Frontiers of Therapeutic Strategies for Brain Tumors: A NeuroMolecular Medicine Special Issue
}

\author{
Vishwanath R. Iyer ${ }^{1}$ John S. Kuo ${ }^{2,3}$ \\ Published online: 7 January 2022 \\ (c) The Author(s), under exclusive licence to Springer Science+Business Media, LLC, part of Springer Nature 2021
}

Primary brain cancers including glioblastoma in adults and various pediatric brain malignancies such as brainstem gliomas and medulloblastomas remain among the most serious causes of morbidity and mortality due to therapeutic resistance and failure of current therapies. Compared to all other cancers, treatment of brain tumors presents unique challenges owing to their relative inaccessibility in the intracranial environment, the vital and irreplaceable nature of the brain and neurological function, the distinct immunological and metabolic characteristics of the brain and the presence of the blood-brain barrier. Primary brain tumors are more refractory to treatment even compared to brain metastases derived from other cancers. Thus, although recent immunotherapies like immune checkpoint blockade are effective in a significant proportion of patients with brain metastases of melanoma, no similar benefit is observed for primary brain tumors.

This NeuroMolecular Medicine special issue entitled "Emerging Frontiers of Therapeutic Strategies for Brain Tumors" is therefore a timely collection of reviews on new frontiers. They highlight multiple approaches that are being developed in the pre-clinical and clinical arena for treatment of brain tumors as well as monitoring therapeutic responses. One stumbling block that needs to be addressed is the limited efficacy of standard immunotherapies for brain tumors. Onate et al. discuss efforts to boost immune responses in brain tumors by combining radiotherapy with tumor-specific

John S. Kuo

john.kuo@cmu.edu.tw

1 Department of Molecular Biosciences, Livestrong Cancer Institutes and Department of Oncology, Dell Medical School, The University of Texas, Austin, TX, USA

2 Department of Neurosurgery, Mulva Clinic for the Neurosciences, Dell Medical School, The University of Texas at Austin, Austin, TX, USA

3 Graduate Institute of Biomedical Sciences, China Medical University, Taichung, Taiwan antibodies, immune checkpoint inhibition and cytokine treatment to achieve an in situ vaccination effect. Although it is most promising in the treatment of brain metastases of other cancers, this approach might also yield benefits for primary brain tumors. Feldman et al. provide an account of adoptive cell immunotherapy to combat glioblastoma, using autologous, genetically modified chimeric antigen receptor (CAR) $\mathrm{T}$ cells. This review highlights trials with second generation CAR T cells directed against different glioblastoma-specific antigens and discusses the unique challenges of applying this approach to brain cancers. Continuing on the theme of improving immunotherapy, Melnick et al. describe novel RNA-based vaccination therapy being developed for glioblastoma. In one method, dendritic cells isolated from the patient are pulsed with mRNA encoding a tumor antigen of choice or with tumor cell mRNA. Then, transfected dendritic cells are re-introduced into the patient where they provoke a $\mathrm{T}$ cell response targeting the tumor cell antigen(s). Alternatively, synthetic nanoparticles loaded with RNA that encode tumor antigens can be administered to patients for targeted expression in dendritic cells, resulting in the stimulation of an anti-tumor $\mathrm{T}$ cell response.

On a different tack, Kukreja et al. focus on ongoing pre-clinical and clinical studies targeting the epigenetic machinery-DNA and histone modifications and noncoding RNA - that is important in regulating gene expression programs in various brain cancers. Here the diversity of epigenetic processes and factors, together with the variety of small molecule inhibitors and miRNA modulators, many of which have shown success in other cancers, provide ample justification for the many ongoing clinical trials of epigenetic targeting agents in brain cancers. Metabolic reprogramming is also a known hallmark of brain cancers and can potentially be exploited for therapy through the use of drugs that disrupt the altered metabolic pathways that are highly utilized by tumor cells and tumor initiating cells. One approach for doing this in gliomas is discussed by $\mathrm{Wu}$ et al. focusing on drugs that inhibit mitochondrial oxidative phosphorylation. 
Appropriate inhibition could potentially target both glioma stem cells as well as tumor cells in distinct ways depending on their differential requirement for this critical metabolic pathway. The contribution of therapeutic resistant cancer stem cells is also examined in detail relative to p53-mediated mechanisms by Treisman et al. for the high morbidity pediatric brain cancer, medulloblastoma. Pivoting from small molecule drugs to bioengineering approaches, Aryal and Porter cover the use of focused ultrasound as a treatment for brain tumors through a number of applications such as opening of the blood-brain barrier, thermal ablation of tumors, hyperthermia and mechanical disruption for improved drug delivery, as well as debulking tumors. Focused ultrasound is clinically approved for certain neurological conditions and is under evaluation in pre-clinical studies and clinical trials for brain tumors.

Like many cancers, glioblastoma shows higher incidence and worse outcomes in males. Lee et al. describe the growing evidence that a multitude of mechanisms give rise to sexbased differences in immune response, genetic aberrations and the cancer-related microbiome. This review underscores that sex-based differences could have significant implications with regard to the efficacy of immunotherapy in brain cancer and that these differences need to be properly considered for optimal therapeutic strategies. Lastly, the importance of noninvasive methods to monitor tumor progression of brain cancers cannot be overstated. Mathios et al. provide a broad overview of newer imaging modalities that show promise in this regard, in particular, the development of radiomics, a way of algorithmically combining features from MRI, CT and PET imaging. Additionally, they discuss new liquid biopsy assays aimed at detecting circulating tumor cells, cell-free DNA and extracellular vesicles in both blood and cerebrospinal fluid, for monitoring tumor response and pseudo-progression after treatment, which is particularly relevant during immunotherapy response in brain cancers.

The collection of papers in this special issue highlights the promise of several emerging therapeutic and monitoring strategies for the deadly collection of neurological diseases represented by brain cancers. The described therapeutic strategies and approaches provide optimism that more advances are forthcoming to resolve some of the current formidable challenges in treating brain tumors and improve clinical outcomes for many future patients.

Publisher's Note Springer Nature remains neutral with regard to jurisdictional claims in published maps and institutional affiliations. 